\title{
Optical properties of zinc titanate perovskite prepared by reactive $R F$ sputtering
}

\author{
Jarmila Müllerová*, Pavol Šutta ${ }^{* *}$, Rostislav Medlín ${ }^{* *}$, \\ Marie Netrvalová**, Petr Novák ${ }^{* *}$
}

\begin{abstract}
In this paper we report results from optical transmittance spectroscopy complemented with data on structure from XRD measurements to determine optical properties of a series of $\mathrm{ZnTiO}_{3}$ perovskite thin films deposited on glass by reactive magnetron co-sputtering. The members of the series differ by the titanium content that was revealed as an origin of the changes not only in structure but also in dispersive optical properties. Low porosity has been discovered and calculated using the Bruggeman effective medium approximation. An apparent blue-shift of the optical band gap energies with increasing titanium content was observed. The observed band gap engineering is a good prospective for eg optoelectronic and photocatalytic applications of $\mathrm{ZnTiO}_{3}$

K e y w o r d s: perovskite, optical band gap, Taucs plot, refractive index, absorption coefficient, effective medium, voids
\end{abstract}

\section{Introduction}

Perovskites are materials of special stoichiometry $\mathrm{ABX}_{3}$ with $\mathrm{X}$ being anions, typically oxygen or halogens, B usually transition metals elements, eg Ti cations, and $\mathrm{A}$ great metal cations, usually alkaline earth or rare earth elements, eg Ca, Zn [1, 2]. Oxide mineral $\mathrm{CaTiO}_{3}$ was discovered in 1839 in the Ural Mountains and named for Russian mineralogist L. A. Perovski. Synthetic perovskites such as $\mathrm{KNbO}_{3}, \mathrm{KBNNO}$, and organic-inorganic $\mathrm{CH}_{3} \mathrm{NH}_{3} \mathrm{PbI}_{3}$ have become famous recently as prospective materials for solar cells with remarkable efficiency progress from the pioneer efficiency of $2 \%$ in 2009 to the efficiency jump of $22.1 \%$ in March 2016 [2,3].

Perovskites show a great diversity of structural modifications leading to a variety of properties and practical applications. The ideal cubic crystal structure of $\mathrm{ABX}_{3}$ is rare and more often is distorted with reduced symmetry $[1,4]$. Perovskite type mixed-metal titanium-based oxides $\mathrm{A}^{I I} \mathrm{Ti}^{I V} \mathrm{O}_{3}$ manifest unique physical and chemical properties. Using $\mathrm{Pb}, \mathrm{Sr}, \mathrm{Ba}, \mathrm{Zn}$ or Fe as metal cations an outstanding potential in dielectric and optoelectronic applications arises. A great attention has been paid to hybrid organic-inorganic perovskites. Among perovskite applications also fuel cells, gas sensors, memory devices, photocatalysis, non-linear optics, solar energy conversion for water splitting and decontamination must be mentioned $[5,6,7]$.

Zinc titanate $\mathrm{ZnTiO}_{3}$ has been reported to have remarkable mechanical, optical and insulating properties $[8,9]$. Due to high photocatalytic activity it has caught high attention [10]. $\mathrm{ZnO}$ and $\mathrm{TiO}_{2}$ are known as wideband semiconductors. Enormous increase of applications based on $\mathrm{ZnO}$ and $\mathrm{TiO}_{2}$ has been caused not only by the improvements of their intrinsic properties but also by the achievements of transition metal doping and mixed oxides formations. Coupling $\mathrm{TiO}_{2}$ with $\mathrm{ZnO}$ leading to Zn-Ti-O ternary oxides offers even more efficient electronhole pair separation under illumination and contributes to the applications in optoelectronic devices including photovoltaic and dye sensitized solar cells, flat panel displays, photodetectors, sensors and light emitting diodes.

$\mathrm{Zn}$-Ti-O ternary compounds comprise $\alpha-\mathrm{Zn}_{2} \mathrm{TiO}_{4}$ (cubic spinel crystal structure), zinc titanate $\left(\mathrm{ZnTiO}_{3}\right.$ either cubic spinel or hexagonal perovskite structure) and $\mathrm{Zn}_{2} \mathrm{Ti}_{3} \mathrm{O}_{8}$ (cubic spinel structure). They are still under extensive studies to fully understand how the incorporation of titanium takes place and how influences the physical properties. Moreover the phase transformation and its effect on optical and dielectric properties of $\mathrm{ZnTiO}_{3}$ thin films are still under considerable debate $[11,12,13]$ and deserve further experimental consideration.

In this paper we report the analysis of optical properties of a series of $\mathrm{ZnTiO}_{3}$ thin films deposited by reactive magnetron co-sputtering affected by different titanium content. Dispersive absorption coefficients and refractive indices were determined from UV Vis transmission spectra. Obvious blue-shift of the absorption edge due to the increasing titanium content was observed. The observed changes are discussed with relation to the structure, microstructure and phase composition of the samples.

* Institute of Aurel Stodola, University of Žilina, Nálepku 1390, 03101 Liptovský Mikuláš, Slovakia, mullerova@lm.uniza.sk, ** New Technologies - Research Centre, University of West Bohemia Univerzitn 8, 30614 Plzeň, Czech Republic 


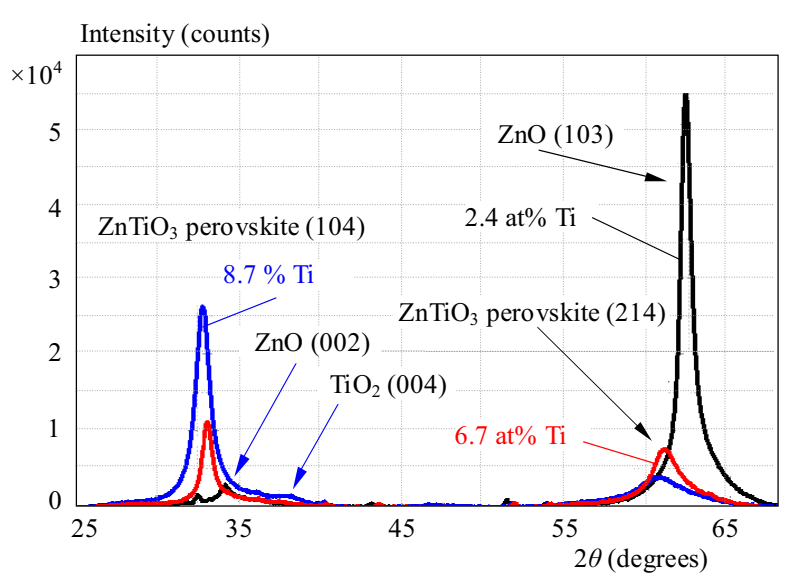

Fig. 1. XRD patterns of three samples scanned in the asymmetric experiment geometry showing the polycrystalline character of the samples

\section{Experimental}

A series of ternary Zn-Ti-O thin films was prepared by reactive magnetron co-sputtering in reactive mode using $\mathrm{RF}$ magnetron unit equipped with pure zinc target and

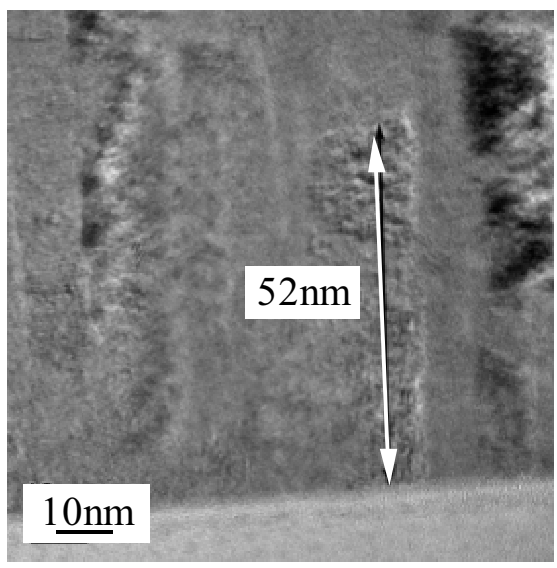

DC magnetron unit equipped with pure $99.99 \%$ titanium target.

The power ratio was $P_{\mathrm{Ti}} /\left(P_{\mathrm{Ti}}+P_{\mathrm{Zn}}\right)$, where $P_{\mathrm{Ti}}$ is the power delivered to titanium target and $P_{\mathrm{Zn}}$ is the power delivered to zinc target. The series contains seven members deposited with increasing atomic percentage titanium content. Structure and titanium content was documented by EDS and EELS measurements and confirmed by Auger electron spectrometry depth profiles [14]. The thin films of the thickness of $200-300 \mathrm{~nm}$ were deposited on clean Corning Eagle 2000 glass substrates and on crystalline silicon wafers.

Structural properties of the films deposited on silicon substrates were analysed by X-ray diffraction (XRD) using an automatic powder diffractometer XPert Pro with $\mathrm{CuK} \alpha$ characteristic radiation $(\lambda=0.154 \mathrm{~nm})$. Two goniometer setups (Bragg-Brentano symmetric $\theta-\theta$, Seemann-Bohlin asymmetric $\omega-2 \theta)$ were used. XRD patterns were collected from 25 to 65 degrees $(\theta-\theta)$ and from 25 to 75 degrees $(\omega-2 \theta)$, [15]. In order to identify the phase composition and basic microstructure parameters (crystallite sizes and micro-strains) of the films

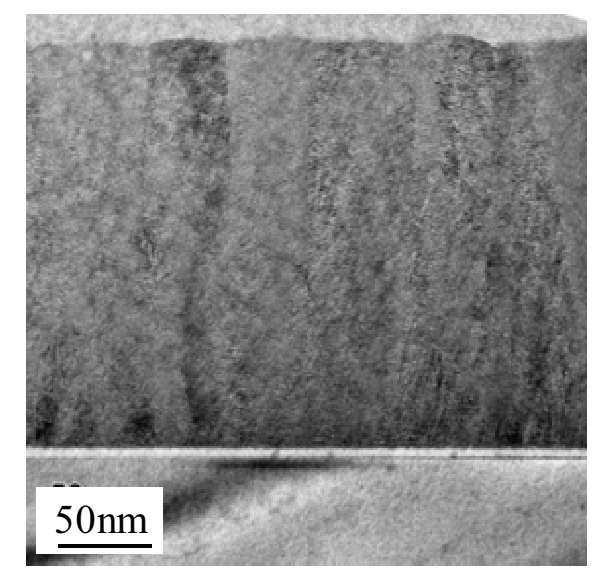

Fig. 2. HR - TEM images of the samples of 2.4 at $\%$ of titanium (left) and 6.7 at\% of titanium (right)

Table 1. XRD identification of the structure of the samples

\begin{tabular}{llccc}
\hline $\begin{array}{c}\text { Sample: } \\
\mathrm{Ti} \\
(\text { at\% })\end{array}$ & Structure & $\begin{array}{c}\text { Phase } \\
\text { identification }\end{array}$ & $\begin{array}{c}\text { Prevailing } \\
\text { crystallite } \\
\text { size }(\mathrm{nm})\end{array}$ & $\begin{array}{c}\text { Thickness } \\
(\mathrm{nm})\end{array}$ \\
\hline 0 & polycrystal & $(002) \mathrm{ZnO}$ & 84 & 235 \\
1.1 & polycrystal & $(002) \mathrm{ZnO}$ & 60 & 210 \\
2.4 & polycrystal & $(002),(101) \mathrm{ZnO}$, bimodal & 52 & 210 \\
5.2 & polycrystal & $(104) \mathrm{ZnTiO}_{3},(004) \mathrm{TiO}_{2}$ atanas, bimodal & 40 & 170 \\
6.7 & polycrystal & $(104) \mathrm{ZnTiO}_{3},(004) \mathrm{TiO}_{2}$ atanas, bimodal & 48 & 225 \\
8.7 & polycrystal & $(104) \mathrm{ZnTiO}_{3},(004) \mathrm{TiO}_{2}$ atanas, bimodal & 19 & 290 \\
12.5 & amorphous & $(104),(110) \mathrm{ZnTiO}_{3}$ overlapped and shifted & & \\
& & due to intersticial oxygen & - & 310 \\
\hline
\end{tabular}

bimodal = bimodal distribution of crystallites 


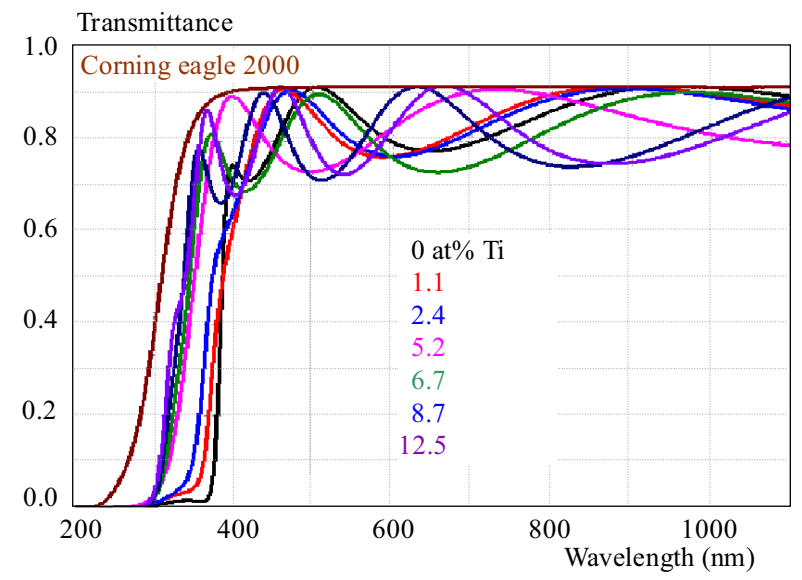

Fig. 3. Transmittance spectra of the samples under study - the legend notifies the titanium content in at\%

line profile analysis of the strongest lines was performed. Crystallite sizes and micro-strains were evaluated by procedures based on the Voigt function [16, 17, 18].

UV VIS spectrophotometry was used to obtain additional microstructural (void volume fraction) and optical parameters of the films (refractive indices, absorption coefficients, optical band gap energies). Dual beam spectrophotometer Specord 210 with air blank reference channel was used to collect UV Vis transmittance spectra of the samples deposited on glass.

\section{Optical properties and structure: Results and discussion}

The analysis of XRD patterns showed that with increasing atomic percentage titanium content the phase composition evolved from $\mathrm{ZnO}$ to $\mathrm{Zn}_{x} \mathrm{Ti}_{1-x} \mathrm{O}_{y}$ and from more to less crystalline structure which was clearly seen from the asymmetry of the diffraction line profiles, Fig. 1. The sample of the highest titanium content was identified as amorphous. With increasing titanium content the evolution of the polycrystalline structure of decreasing crystallite size (except of the amorphous sample of the highest titanium content of 12.5 at\%) and the preferred orientation of crystallites in a certain direction against the surface (Tab. 1) was observed. The thickness was determined from fitting optical transmittance measurements. Moreover, the samples of the intermediate titanium content (from 2.4 to 8.7 at\%) were found to have bimodal crystallite size distribution. Cross-sectional HR-TEM images of the samples of the atomic percentage titanium content of 2.4 and 6.7 confirming the presence of various crystallites are in Fig. 2.

In the optical transmittance spectra (Fig. 3) - the transmittance of the Corning Eagle 2000 glass substrate is indicated - apparent interference fringes are present due to weak absorption of light above the absorption edge. The onset of the material transparency at the wavelengths $>\approx 300 \mathrm{~nm}$ is obviously shifted what indicates the differences in optical band gaps of the samples of different titanium content.

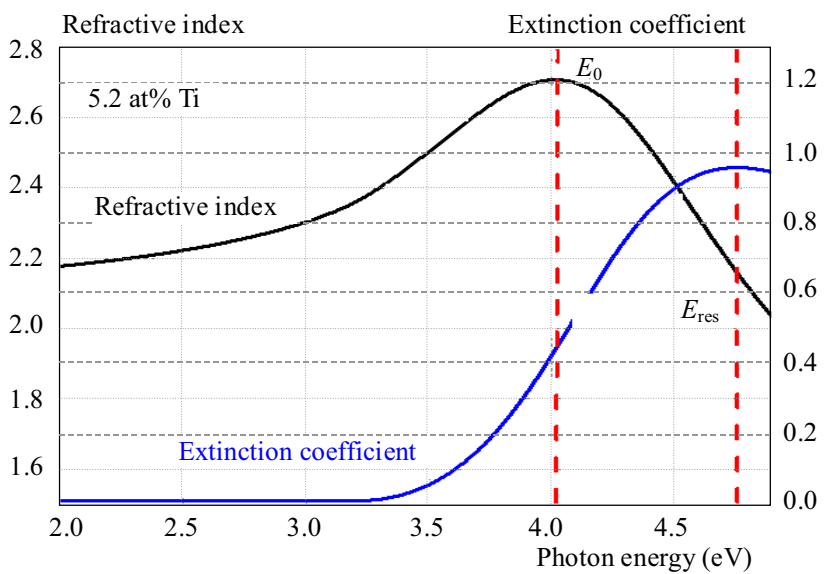

Fig. 4. Photon energy dependent refractive indices and extinction coefficients of the sample of 5.2 at $\%$ of titanium

The transmittance of a homogeneous non-scattering thin film with parallel interfaces deposited on a thick uncoherent substrate is a nonlinear function of the wavelength, the film thickness and the complex refractive indices of the film and the substrate. The spectral refractive index $n$ and the extinction coefficient $k$ are the real and imaginary parts of the complex refractive indices of the film and the substrate. Spectral refractive indices $n$ and extinction coefficients $k$ of the films were extracted from the measured transmittance spectra using a global optimization procedure based on genetic algorithm. The optimization procedure minimized differences between the experimental and theoretical transmittance in the broad spectral region including the region in the vicinity of the absorption edge and enabled to search for the solution with preventing false solutions at local minima. The theoretical transmittance was calculated using the theory in [19] and the Tauc-Lorentz dispersion model for the refractive index and the extinction coefficient [20] that has been currently employed for the parameterization of the optical functions of amorphous materials. Results for $n, k$ are in good agreement with the theoretical calculations [21].

In Fig. 4 the results of $n, k$ versus the photon energy $E_{\text {phot }}=h c / \lambda, \quad(h$ is the Planck's constant, $c$ is the speed of light) related to the wavelength $\lambda$ can be seen for the sample of 5.2 at\% of titanium. The KramersKronig dispersion relation is apparently obeyed. The peak of the refractive index $E_{0}$ is the so-called central energy $E_{0}$ in the Tauc-Lorentz dispersion model, the peak of the extinction coefficient $E_{\text {res }}$ is the resonance energy of the electronic transition. Resulting wavelength dependent refractive indices of all samples are in Fig. 5. Central energies are clearly blue-shifted with growing titanium content.

Extrapolating spectral refractive indices to the infrared $i e$ to the non-absorbing region the so-called refractive index in the long wavelength limit $n_{\infty}$ can be obtained. This parameter is related to the atomic structure and the mass density. The values of $n_{\infty}$ in Tab. 2 show that with the increasing titanium content this parameter starts to decrease after apparent initial growth. 


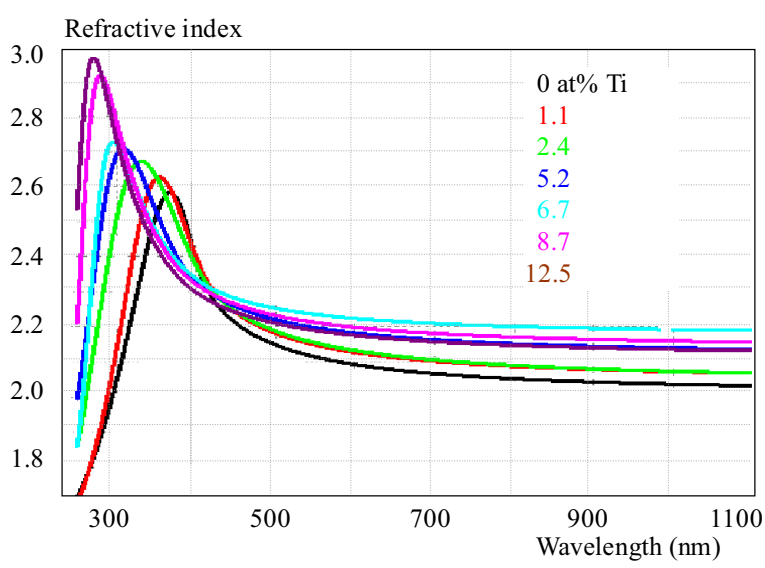

Fig. 5. Refractive indices of the $\mathrm{ZnTiO}_{3}$ samples of different titanium content

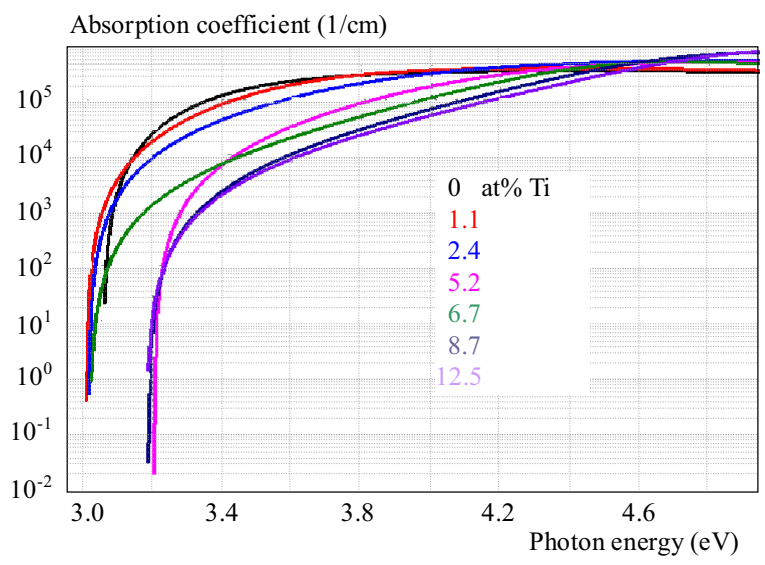

Fig. 7. Absorption coefficients of the $\mathrm{ZnTiO}_{3}$ samples of different titanium content

Fig. 6 shows a somewhat skewed distribution of $n_{\infty}$ as a function of titanium content illustrating this trend. The solid line represents fitting by Gumbel distribution extreme function. This observation indicates that samples of growing titanium content up to $\approx 7$ at $\%$ became denser. Surprisingly this trend stops then and $n_{\infty}$ starts to decrease. This means that the titanium content does not play the single role in the mass density of the samples. We deduce that the same importance originates from the phase composition, structure and crystallite size.

Refractive indices of the samples in the region from 300 $\mathrm{nm}$ up to the central energy apparently increase with increasing titanium content what can be seen in Fig. 3.

As often expected the presence of microvoids may play important role in photovoltaic applications of perovskites. Internal surfaces of voids can be broken by light-induced charge carriers what translates to light-induced defects, $e g$ an oxidation. Thus it is recommended to control the porosity and to keep it as low as possible using proper deposition chemistry. On the other hand porosity is required for forming perovskites for photocatalytic or oxidation reactions [22]. Therefore the determination of the porosity via various effective medium approximations (EMA) dealing with effective properties of a medium with inclusions

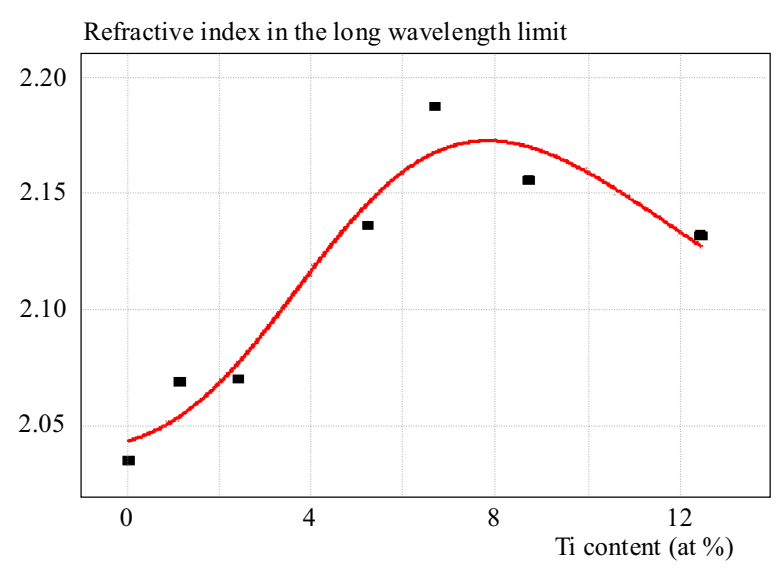

Fig. 6. The refractive index in the long wavelength limit versus the titanium content

$[23,24]$ is evitable. According to EMA a mixture of different materials can be considered as a homogenous medium having an effective refractive index that can be obtained from refractive indices and volume fractions of individual components. Bruggeman EMA (BEMA) [24] is useful in case of aggregate structure filled randomly with a mixture of two or more phases. This approximation represents a realistic model for systems with irregularly shaped particles corresponding well to perovskites with crystalline grains of conical or cylindrical shape.

According to BEMA the following equation is valid for a composite film of several crystalline/amorphous constituents of volume fractions pi $(i=1,2, \ldots)$ and $\mathrm{pv}$ (microvoids) in the non-absorbing region

$$
\begin{aligned}
& p_{1} \frac{n_{1}^{2}-n_{\infty}^{2}}{n_{1}^{2}+2 n_{\infty}^{2}}+p_{2} \frac{n_{2}^{2}-n_{\infty}^{2}}{n_{2}^{2}+2 n_{\infty}^{2}}+ \\
& p_{3} \frac{n_{3}^{2}-n_{\infty}^{2}}{n_{3}^{2}+2 n_{\infty}^{2}}+\ldots+p_{v} \frac{1-n_{\infty}^{2}}{1+2 n_{\infty}^{2}}=0
\end{aligned}
$$

while $\sum_{i} p_{i}+p_{v}=1$. In (1) $n_{i}$ are refractive indices of individual constituents and $n_{v}=1$. The ratios of volume fractions $p_{i} / p_{j}$ of individual constituents $\left(\mathrm{ZnO}, \mathrm{TiO}_{2}\right.$, $\left.\mathrm{ZnTiO}_{3}\right)$ were determined from XRD measurements. The refractive index in the long wavelength limit $n_{\infty}$ can be directly included as the effective medium refractive index to avoid dealing with the complex refractive indices in (1). Results for voids volume fractions $p_{v}$ in Tab. 2 show that the porosity was kept below $10 \%$ reaching a minimum at 6.7 at $\%$ of titanium.

For all optoelectronic applications, fundamental optical absorption is a meaningful issue. From Fig. 3 we can see that the onset of the absorption in the samples is different. Let us define the absorption edge (in eV) simply as the intersection of the linear part of optical transmittance with the horizontal axis at the transmittance equal to zero. As seen the absorption edges (Tab. 2) are well shifted to the blue and ultraviolet region and grow with increasing titanium content. 


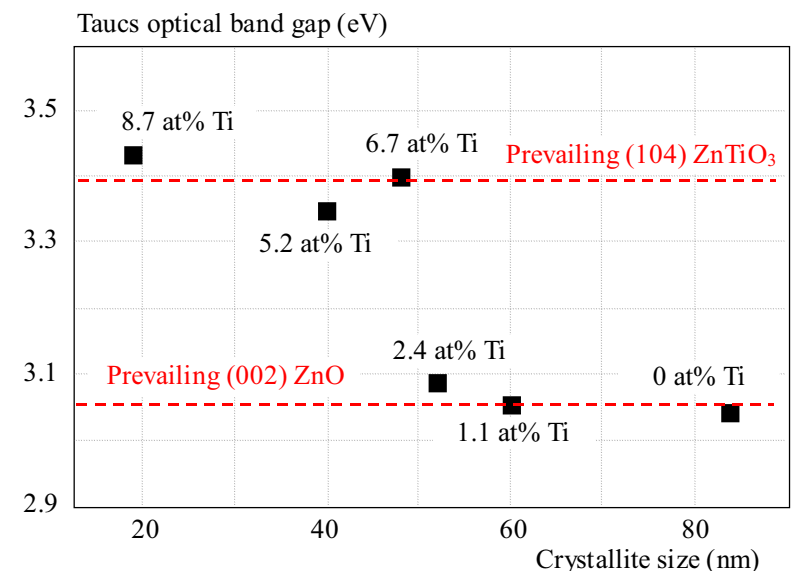

Fig. 8. The relation of the Tauc's optical band gap energies to the crystallite size corresponding to the samples with varying titanium content

The absorption coefficient $\alpha$ is related to the extinction coefficient $\mathrm{k}$ through the relation $\alpha=4 \pi k / \lambda$. Figure 7 demonstrates the changes of the absorption coefficients of samples with varied amount of titanium and therefore various band gap energies as a result of the changing titanium content can be expected. The isoabsorption gaps $E_{04}$ are often used to express the values of photon energy at which the absorption coefficient in the samples achieves the value of $10^{4} \mathrm{~cm}^{-1}$.

Due to disorder in the mixed-phase semiconductors the valence and conduction band edges are extended. Then the band tails are quite blurred with no clear abrupt energy above which photons are absorbed via interband transitions. Therefore several procedures based on variously expressed density of state distributions in the extended states near the valence and conduction bands and the transition matrix elements can be used for calculating the band gap energies. A usual practice is the socalled Tauc's plot originating from the constant transition matrix element squared. The related optical band gaps are determined from the extrapolation of linear parts of Tauc's plots $(\alpha \hbar \omega)^{1 / 2} \propto\left(E_{\mathrm{phot}}-E_{g}^{\mathrm{opt}}\left(E_{g}^{\mathrm{opt}}\right.\right.$ is the Tauc's optical band gap) to the energy axis. The values of Tauc's optical band gaps versus the crystallite size are depicted in Fig. 8. Two groups of values corresponding to prevailing $\mathrm{ZnO}$ at lower titanium content and $\mathrm{ZnTiO}_{3}$ at higher titanium content can be recognized.

Optical band gap energies are summarized in Tab. 2. The results provide a satisfactory mean of comparing optical absorption in the samples. An interesting symptom is that the band gap energies are organized in two groups corresponding to the samples with prevailing $\mathrm{ZnO}$ and $\mathrm{ZnTiO}_{3}$ (Fig. 8). Moreover it is seen that optical band gap energies reflect well the increasing titanium content and the changing structure and are blue-shifted (towards higher energies $i e$ towards smaller wavelengths). Furthermore we can conclude similarly as in [25] that a gradual increase of optical band gap energies occurs with the decrease of the crystallite size (Tab. 2, Fig. 8). This modification of the absorption spectrum can be linked to the size effect. Although the size effect is more recognizable at the crystallite size below $10 \mathrm{~nm}$, it occurs even at the crystallites of less than $100 \mathrm{~nm}$ and comprises not only quantum size effect but also the effects of grain boundaries on thin film properties.

\section{Conclusions}

Extensive optical characterization of $\mathrm{ZnTiO}_{3}$ thin films based on the transmittance spectra analysis in UV Vis region complemented with knowledge on structure from XRD has been successful to show that the films with growing titanium content evolve from polycrystalline to amorphous structure with increasing optical band gap energies. The spectral dependencies of refractive indices and absorption coefficients were determined to indicate changes linked to the film structure and the density. The void volume fractions have been determined using the effective medium approximation to find conditions for obtaining samples with predictable void fraction. The analysis demonstrated that the optical band gaps increase with increasing titanium content that corresponds to decreasing crystallite size. From our analysis it implies that the tuning of the optical band gap with the sample composition, ie the titanium content is also possible.

Table 2. Optical properties of the $\mathrm{ZnTiO}_{3}$ samples of different atomic percentage content of titanium

\begin{tabular}{lcccccc}
\hline $\begin{array}{l}\text { Sample: } \\
\text { Sample: }\end{array}$ & $n_{\infty}$ & $\begin{array}{c}p_{v} \\
(\%)\end{array}$ & $\begin{array}{c}\text { Absorption } \\
\text { edge } \\
(\mathrm{eV})\end{array}$ & $\begin{array}{c}\text { Central } \\
\text { energy } \\
(\mathrm{eV})\end{array}$ & $\begin{array}{c}\text { Tauc's } \\
E_{g} \\
(\mathrm{eV})\end{array}$ & $\begin{array}{c}E_{04} \\
(\mathrm{eV})\end{array}$ \\
\hline 0 & 2.028 & 9.3 & 3.05 & 3.38 & 3.04 & 3.14 \\
1.1 & 2.064 & 8.1 & 3.01 & 3.5 & 3.05 & 3.15 \\
2.4 & 2.064 & 8.1 & 3.03 & 3.75 & 3.08 & 3.21 \\
5.2 & 2.133 & 2.3 & 3.2 & 4.01 & 3.35 & 3.44 \\
6.7 & 2.187 & 0.5 & 3.03 & 4.19 & 3.4 & 3.46 \\
8.7 & 2.153 & 1.5 & 3.18 & 4.43 & 3.43 & 3.6 \\
12.5 & 2.128 & 1.9 & 3.2 & 4.58 & 3.62 & 3.65 \\
\hline
\end{tabular}




\section{Acknowledgements}

The results were developed within the CENTEM project, reg. no. CZ.1.05/2.1.00/03.0088, co-funded by the ERDF as part of the Ministry of Education, Youth and Sports OP RDI programme and, in the followup sustainability stage, supported through CENTEM+ (LO1402) by financial means from the Ministry of Education, Youth and Sports under the National Sustainability Programme I. The article was partly created with the support of the Ministry of Education, Science, Research and Sport of the Slovak Republic within the Research and Development Operational Programme for the project "University Science Park of STU Bratislava", ITMS 26240220084, co-funded by the European Regional Development Fund. This work was partially supported by the Slovak Grant Agency under the project VEGA 2/0076/15 and by the Slovak Research and Development Agency under the projects APVV-0888-12 and APVV15-0152.

\section{REFERENCES}

[1] R. J. D. Tilley, Perovskites: Structure-Property relationships, John Wiley \& Sons Ltd., 2016, ISBN 9781118935668.

[2] N. K. Elumalai, Md A. Mahmud, D. Wang and A. Uddin, "Perovskite solar cells: Progress and advancements, Energies", vol. 9, 861, 2016, pp. 1-20.

[3] NREL Best Research-Cell Efficiencies.Available online: http:// www. nrel.gov/ncpv/images/ efficiency ${ }_{c}$ hart.jpg (accessed on 22 February 2016).

[4] M. W. Lufaso and P. M. Woodward, "Prediction of the crystal structures of perovskites using the software program SPuDS", Acta Cryst. , vol. B57, 2001, pp. 725-738.

[5] M. Petrović, V. Chellappan and S. Ramakrishna, "Perovskites: Solar cells \& engineering applications - materials and device developments, ", Solar Energy, vol. , 122, 2015, pp. 678-699.

[6] S. Brittman, G. W. P. Adhyaksa and E. C. Garnett, "The expanding world of hybrid perovskites: materials properties and emerging applications", MRS Commun., vol. 5, 2015, pp. 7-26.

[7] Q. Chen, N. De Marco, Y. M. Yang, T.-B. Song, C.-C. Chen, H. Zhao, Z. Hong, H. Zhou and Y. Yang, "Under the spotlight: The organic-inorganic hybrid halide perovskite for optoelectronic applications", Nano Today, vol. 10, 2015, pp. 355-396.

[8] Q. -J. Liu, N. -C. Zhang, F. -S. Liu, H. -Y. Wang and Z. -T. Liu, "Theoretical study of structural, elastic, electronic properties, and dispersion of optical functions of hexagonal $\mathrm{ZnTiO}_{3}$ ", Phys. Status Solidi B, 250, 9, 2013, pp. 1810-1815.

[9] Z. Ali, S. Ali, I. Ahmad, I. Khan and H. A. R. Aliabad, "Structural and optoelectronic properties of the zinc titanate perovskite and spinel by modified Becke-Johnson potential", Physica B, vol. 420, 2013, pp. 54-57.

[10] X. Yan, C.-L. Zhao, Y.-L, Zhou, Z.-J. Wu, J.-M. Yuan and W.-S. Li, "Synthesis and characterization of $\mathrm{ZnTiO}_{3}$ with high photocatalytic activity", Trans. Nonferrous Met. Soc. China, vol. 25, 2015, pp. 2272-2278.

[11] P. K. Jain, M. Salim and D. Kaur, "Effect of phase transformation on optical and dielectric properties of pulsed laser deposited $\mathrm{ZnTiO}_{3}$ thin films", Superlattices and Microstructures, vol. 92, 2016, pp. 308-315.

[12] Y.-C. Lee, Y.-L. Huang, W.-H. Lee and F.-S. Shieu, "Formation and transformation of $\mathrm{ZnTiO}_{3}$ prepared by sputtering process", Thin Solid Films, vol. 518, 2010, pp. 7366-7371.

[13] I. Caretti, M. Yuste, R. Torres, O. Sánchez, I. Jiménez, and R. Escobar Galindo, "Coordination chemistry of titanium and zinc $\mathrm{Ti}_{(1-x)} \mathrm{Zn}_{2 x} \mathrm{O}_{2}(0 \leq x \leq 1)$ ultrathin films grown by DC reactive magnetron sputtering", RSC Advances, vol. 2, 2012, pp. 2696-2699.

14] L'. Vančo, Slovak University of Technology, Bratislava, personal communication, 2016.

15] R. Medlín, P. Šutta, M. Netrvalová and P. Novák, "Investigation of structural changes of $\mathrm{ZnO}$ :Ti thin films prepared by $\mathrm{RF}$ sputtering", European Microscopy Congress 2016: Proceedings, 2016, pp. 655-656.

16] R. Siddeswaran, P. Šutta, P. Novák, A. Hendrych and O. Životsky, "In-situ X-ray diffraction studies and magneto-optic Kerr effect on RF sputtered thin films of $\mathrm{BaTiO}_{3}$ and $\mathrm{Co}$, $\mathrm{Nb}$ co-doped $\mathrm{BaTiO}_{3}$ ", Ceramics International, vol. 42, 2016, pp. 3882-3887.

[17] J. I. Langford, "Diffraction line profile analysis procedure for studying microstructure", Proc. of the III School on X-ray Diffraction from Polycrystalline Materials, University of Trento (Italy) 16-18 February 1994, pp. 69-82.ISBN 88-86135-25-4.

18] M. Zeman, G. van, F. D. Elzakker, P. Tichelaar and P. Šutta, "Structural properties of amorphous silicon prepared from hydrogen-diluted silane", Philosophical Magazine, vol. 89, 2009, pp. $2435-2448$.

[19] I. Chambouleyron, J. M. Martinez, A. C. Moretti and M. Mulato, "Retrieval of optical constants and thickness of thin films from transmission spectra", Appl. Optics, vol. 36, 1997, pp. 8238-8247.

[20] G. E. Jellison Jr and F. A. Modine, "Parametrization of the optical functions of amorphous materials the interband region, ", Appl.Phys.Lett., vol. 69, 1996, pp. 371-373 and erratum Appl.Phys.Lett., vol. 69, 1996, p. 2137.

[21] X.-C. Zhang, C.-M. Fan, Z.-H. Liang and P.-D. Han, "Electronic structures and optical properties of ilmenite-type hexagonal $\mathrm{ZnTiO}_{3}$ ", Acta Phys.-Chim.Sin., vol. 27, 2011, pp. 47-51.

[22] G. Battilana, A. Bottino, G. Capannelli and P. Nanni, "The preparation of porous perovskite membranes using $\mathrm{BaTiO}_{3}$ nanopowders", J.Mat.Sci., vol. 37, 2002, pp. 4343-4347.

[23] T. C. Choy Effective medium theory - principles and applications, Oxford University Press 1999.

[24] D. A. G. Bruggeman, "Berechnung verschiedener physikalischer Konstanten von heterogenen Systemen", Ann.Phys., vol. 24, 1935, pp. 636-679.

25] L. Budigi, M. R. Nasina, K. Shaik and S. Amaravadi, "Structural and optical properties of zinc titanates synthesized by precipitation method", J. Chem. Sci., vol. 127, 2015, pp. 509-518.

Received 23 April 2017

Jarmila Müllerová (Prof, $\mathrm{RNDr}, \mathrm{PhD}$ ) received her $\mathrm{PhD}$ Degree in quantum electronics and non-linear optics from the Comenius University, Bratislava, Slovakia. Since 2003 she has been with the University of Žilina, Slovakia. Currently she is a Full Professor in Electrotechnologies and Materials at the Institute of Aurel Stodola, the Faculty of Electrical Engineering. Her ongoing research interests include optical properties of solids, thin films optics, optics of multilayered structures and interfaces. Her attention is also focused on wavelengthsensitive devices for the physical layer of all-optical communication networks, namely for WDM applications.

Pavol Šutta (Assoc Prof, RNDr, PhD) received his $\mathrm{PhD}$ degree in physics of condensed matter and acoustic from the Comenius University, Bratislava, Slovakia. Since 2003 he has been with the New Technologies - Research Centre of the University of West Bohemia, the Czech Republic. He is active in crystallography, solid state physics and X-ray diffraction. In the course of many years he has devoted himself to X-ray 
diffraction analysis of the real structure of the dielectric, semiconductive and metallic thin films prepared by high vacuum evaporation, CVD techniques, diode and magnetron sputtering to serve as solar cells, sensors, micro-sensors and microactuators as well as bulk materials prepared by sintering and powder materials.

Rostislav Medlín (Ing, PhD) received his $\mathrm{PhD}$ degree in physics of plasma and thin films from the University of West Bohemia, Pilsen, the Czech Republic. Since 2002 he has been with the New Technologies Research Centre University of West Bohemia, Czech Republic. He is active in transmission and scanning electron microscopy including electron energy loss spectroscopy, energy and wavelength dispersive spectroscopy and electron diffraction. In the course of many years he devotes himself to preparation and high resolution and diffraction analysis of materials including thin films in transmission microscopy, element analysis of thin films and EELS analysis.
Marie Netrvalová (Ing, $\mathrm{PhD}$ ) studied at the Department of Material Science and Technology, Faculty of Mechanical Engineering at University of West Bohemia in Pilsen, the Czech Republic. Here she received her Ing and PhD degrees. Since February 2007 she has been working at the New Technologies - Research Centre, University of West Bohemia in Pilsen, the Czech Republic. She is focused on optical spectroscopies (UV-Vis spectroscopy and spectroscopic ellipsometry) and vibration spectroscopies (infrared and Raman spectroscopy) on thin films for photovoltaics, photonics and micro-system devices.

Petr Novák (Ing, PhD) is a postdoctoral researcher at the University of West Bohemia. He obtained his Master's degree in 2007 and completed a $\mathrm{PhD}$ in plasma physics and physics of thin films in 2012 at the University of West Bohemia. Following the completion of his $\mathrm{PhD}$ he moved to the New Technologies - Research Centre. His research interests include magnetron sputtering, solid phase crystallization and applications of sputtered thin films for devices such as piezoelectric energy harvesters and photovoltaics. 